\title{
Ultraviolet Properties of Maximal Supergravity
}

\author{
Michael B. Green \\ Department of Applied Mathematics and Theoretical Physics, Wilberforce Road, Cambridge CB3 OWA, United Kingdom \\ Jorge G. Russo \\ Institució Catalana de Recerca i Estudis Avançats (ICREA), University of Barcelona, Avenida Diagonal 647, Barcelona 08028, Spain \\ Pierre Vanhove \\ Service de Physique Théorique, CEA/Saclay, F-91191 Gif-sur-Yvette, France
}

(Received 18 January 2007; published 28 March 2007)

\begin{abstract}
We argue that recent results in string perturbation theory indicate that the four-graviton amplitude of four-dimensional $N=8$ supergravity might be ultraviolet finite up to eight loops. We similarly argue that the $h$-loop $M$-graviton amplitude might be finite for $h<7+M / 2$.
\end{abstract}

DOI: 10.1103/PhysRevLett.98.131602

PACS numbers: 11.25.Db, 04.65.+e

Maximally extended supergravity has for a long time held a privileged position among supersymmetric field theories. Its four-dimensional incarnation as $N=8$ supergravity [1] initially raised the hope of a perturbatively finite quantum theory of gravity while its origin in $N=1$ elevendimensional supergravity [2] provided the impetus for the subsequent development of $M$ theory, or the nonperturbative completion of string theory. Maximal supergravities in various dimensions arise as special limits within type II string theory, which is free of ultraviolet divergences. However, the fact that higher-dimensional maximal supergravity is not renormalizable means that it cannot be quantized in any conventional manner in isolation from string theory.

Another well-known problem with theories such as type II supergravity is that it is notoriously difficult to analyze the constraints implied by maximal supersymmetry in a fully covariant manner since there is no practical off-shell formalism that makes manifest the full supersymmetry. For example, it has not yet been possible to determine the extent to which supersymmetry protects operators against perturbative corrections. However, a number of indirect arguments suggest that such protection may be far greater than earlier estimates might suggest. For example, a certain amount of evidence has accumulated over the past few years that the sum of the Feynman diagrams of maximal supergravity at a given number of loops may be less ultraviolet divergent than expected [3]. This is largely based on uncovering fascinating connections with the diagrams of $N=4$ Yang-Mills theory, which are known to be ultraviolet finite in four dimensions. A different approach is to study implications of string/ $M$-theory duality for the scattering amplitudes of type II supergravity. In Ref. [4] we considered the $L$-loop Feynman diagrams of the fourgraviton scattering amplitude in 11-dimensional supergravity compactified on a two-torus and its string theory interpretation. The lack of information about the shortdistance structure of $M$ theory is reflected by the non- renormalizability of supergravity and this ignorance was parametrized by an unlimited number of unknown coefficients of counterterms. Nevertheless, requiring the structure of the amplitude to be consistent with string theory led to interesting constraints. Among these were strong nonrenormalization conditions in the ten-dimensional type IIA string theory limit - where the 11-dimensional theory is compactified on a circle of radius $R_{11}$. This condition followed from dimensional analysis together with the fact that the string coupling constant is given by $e^{\phi}=$ $R_{11}^{3 / 2}$, where $\phi$ is the dilaton. These conditions imply that the genus- $h$ four-graviton amplitude has a low-energy limit that begins with a power of $\mathcal{S}^{(h)} R^{4}$, where $\mathcal{S}^{(h)}$ is a symmetric monomial of power $h$ made out of the Mandelstam invariants $s, t$, and $u$. This was interpreted as an indication that the $h$-loop amplitude obtained in the low-energy supergravity limit has milder ultraviolet behavior than naive expectations and four-dimensional $N=8$ supergravity might be free of ultraviolet divergences.

However, the arguments of [4] were rather indirect. Here we will proceed more directly and more conservatively by using perturbative string theory as a regulator of the ultraviolet divergences of supergravity. We will see that the nonrenormalization conditions of perturbative type II string theory obtained by Berkovits [5], which are weaker than those proposed in [4], point to the possible absence of ultraviolet divergences in the four-graviton amplitude of four-dimensional $N=8$ supergravity amplitude up to eight loops.

We begin by noting that the $h$-loop contribution to the four-graviton amplitude in ten-dimensional string theory has the form

$$
A_{4}^{h}=\alpha^{\prime \beta_{h}-1} e^{2(h-1) \phi} \sum_{i} \mathcal{S}_{i}^{\left(\beta_{h}\right)} I_{i}^{(h)}\left(\alpha^{\prime} s, \alpha^{\prime} t, \alpha^{\prime} u\right) R^{4},
$$

where $R$ is the Weyl curvature and $\mathcal{S}_{i}^{\left(\beta_{h}\right)}$ are monomials of power $\beta_{h}$ in the Mandelstam invariants $s, t$, and $u$. Explicit 
one-loop [6] and two-loop [3] calculations show that $\beta_{1}=$ 0 and $\beta_{2}=2$. In fact, recent perturbative superstring calculations [5] determine that $\beta_{h}=h$ up to five loops $(h=$ $5)$. The less direct arguments of [4] make use of string/ $M$-theory dualities to argue that $\beta_{h}=h$ might hold to all orders. In the following we will not specify the value of $\beta_{h}$ until we need to. The functions $I_{i}^{(h)}$ are given by integrals over the moduli space of the $h$-loop string world-sheet. The number of such terms depends on the genus. For example, at two loops $(h=2)$ there are three terms,

$$
\begin{aligned}
\sum_{i=1}^{3} \mathcal{S}_{i}^{(2)} I_{i}^{(2)}\left(\alpha^{\prime} s, \alpha^{\prime} t, \alpha^{\prime} u\right)= & s^{2} I_{1}^{(2)}\left(\alpha^{\prime} s, \alpha^{\prime} t, \alpha^{\prime} u\right) \\
& +t^{2} I_{2}^{(2)}\left(\alpha^{\prime} s, \alpha^{\prime} t, \alpha^{\prime} u\right) \\
& +u^{2} I_{3}^{(2)}\left(\alpha^{\prime} s, \alpha^{\prime} t, \alpha^{\prime} u\right) .
\end{aligned}
$$

The detailed evaluation of the functions $I_{i}^{(h)}$ for $h>2$ is a daunting task but here we will only be concerned with the general structure of the amplitude.

We wish to consider the low-energy field theory limit of $A_{4}^{h}$ obtained by expanding the scalar functions $I_{i}^{(h)}$ in the limit $\alpha^{\prime} \rightarrow 0$ while holding the ten-dimensional Newton coupling $\kappa_{(10)}^{2}=\alpha^{\prime 4} e^{2 \phi}$ fixed. Since $I_{i}^{(h)}$, which is an integral over world-sheet moduli, does not have poles in $s, t$, $u$ [5] its low-energy expansion starts with a constant or logarithmic term. At low energies the amplitude (1) therefore takes the symbolic form

$$
A_{4}^{h} \sim \kappa_{(10)}^{2(h-1)} \alpha^{\prime 3-4 h+\beta_{h}} \mathcal{S}^{\left(\beta_{h}\right)}\left[1+O\left(\alpha^{\prime} s\right)\right] R^{4},
$$

where we have not kept track of possible factors that are logarithmic in the Mandelstam invariants but which are, in principle, defined precisely by evaluating the amplitude. The expression (1) is finite in ten-dimensional string theory due to the presence of the string length that provides an ultraviolet cutoff. Indeed, the divergence of the expression (3) in the low-energy limit, $\alpha^{\prime} \rightarrow 0$, translates into the ultraviolet divergence of the sum of all the contributions to the supergravity $h$-loop amplitude. After interpreting the inverse string length as an ultraviolet momentum cutoff $\Lambda \sim \sqrt{\alpha^{\prime-1}}$ (3) becomes

$$
A_{4}^{h} \sim \kappa_{(10)}^{2(h-1)} \Lambda^{8 h-6-2 \beta_{h}} \mathcal{S}^{\left(\beta_{h}\right)}\left[1+O\left(\alpha^{\prime} s\right)\right] R^{4} .
$$

So the presence of the prefactor $\mathcal{S}^{\left(\beta_{h}\right)} R^{4}$ means that the leading divergences $\Lambda^{8 h+2}$ of individual $h$-loop Feynman diagrams cancel and the ultraviolet divergence of the sum of diagrams is reduced by a factor of $\Lambda^{-8-2 \beta_{h}}$.

We are interested in the maximal supergravity limit in lower dimensions so we will consider compactifying the string loop amplitude on a $(10-d)$ torus (with the external momenta and polarizations oriented in the $d$ noncompact directions). Now consider the low-energy limit $\alpha^{\prime} \rightarrow 0$ with the radii of the torus proportional to $\sqrt{\alpha^{\prime}}$, so that all the massive Kaluza-Klein states, winding-number states, and excited string states decouple. This leads to an expression for the $h$-loop supergravity amplitude in $d$ dimensions with cutoff $\Lambda_{d}$. For example, for a square torus with all radii equal to $r \sqrt{\alpha^{\prime}}$, the expression for $A_{4}^{h}$ behaves as $\kappa_{(d)}^{2(h-1)} \Lambda_{d}^{(d-2) h-2 \beta_{h}-6}$, where the $d$-dimensional Newton constant, given by $\kappa_{(d)}^{2}=\alpha^{\prime(d-2) / 2} e^{2 \phi} r^{d-10}$, is held fixed. Consider the low-energy limit in a dimension $d$ for which the power of $\Lambda_{d}$ is positive, so that $(d-2) h>2 \beta_{h}+6>$ 0 . We will make a "smoothness assumption" that this power of $\Lambda_{d}$ does not increase in the process of taking the low-energy limit. Although this sounds plausible, it remains unproven and would fail if the leading low-energy behavior of the function $I_{i}^{(h)}(s, t, u)$ in the compactified theory were an inverse power of $s, t$, or $u$. With this assumption it follows that ultraviolet divergences are absent in dimensions for which

$$
d<2+\frac{2 \beta_{h}+6}{h} .
$$

In dimensions that satisfy this bound the expression (4) is ill defined since it contains a negative power of the cutoff, which vanishes, whereas finite and infrared divergent terms that are nonvanishing are not exhibited. In this case the negative power of $\Lambda_{d}$ is replaced by a negative power of $s$, $t$, and $u$ of dimension $[s]^{(d-2) h / 2-\beta_{h}-3}$, together with possible logarithmic factors. Infrared divergences arise for dimensions $d \leq 4$ and presumably sum up in the usual fashion to cancel the divergences due to multiple soft graviton emission [7]. These features of the field theory limit are seen explicitly in the compactified one-loop $(h=$ 1) amplitude, which has $\beta_{1}=0$ [8]. In that case the amplitude has the form of a prefactor of $R^{4}$ multiplying a $\varphi^{3}$ scalar field theory box diagram. This is ultraviolet divergent when $d \geq 8$, and finite for $4<d<8$. The lowenergy limit of the two-loop $(h=2)$ string theory expression reduces to the supergravity two-loop amplitude which was considered in detail in [3,9]. There it was shown that a power of $s^{2}, t^{2}$, or $u^{2}$ factors out of the sum of all supergravity Feynman diagrams, so that $\beta_{2}=2$. In this case the sum of Feynman diagrams has the form of prefactor $s^{2} R^{4}$ multiplying the sum of planar and nonplanar $s$-channel double box diagrams of $\varphi^{3}$ scalar field theory, together with corresponding $t$-channel and $u$-channel terms. More recently the fact that $\beta_{2}=2$ has been confirmed by explicit two-loop calculations in string theory [10]. In this case ultraviolet divergences arise when $d \geq 7$ and the amplitude is finite for $4<d<7$.

The value of $\beta_{h}$ for $h>2$ has not been established from direct supergravity calculations beyond two loops, but it is strongly suspected that $\beta_{h} \geq 2$ for $h>2$. Furthermore, in contrast to the $h=1$ and $h=2$ cases, the sum of Feynman diagrams for $h>2$ is unlikely to reduce to a prefactor 
multiplying diagrams of $\varphi^{3}$ scalar field theory. That would require $\beta_{h}=2(h-1)$, which would lead to finiteness in $d<6$ dimensions (for $h=3$ it would also contradict the presence of a three-loop term in $D^{6} R^{4}$ found in [11]). Using the value $\beta_{h}=2$ (the least possible value) in (5) leads to the absence of ultraviolet divergences when

$$
d<2+10 / h, \quad h>1,
$$

which appeared in [3]. This shows that the first ultraviolet divergence in four dimensions cannot arise until at least five loops.

The full extent to which the four-graviton amplitude is protected from ultraviolet divergences should become clearer with a more complete understanding of the constraints implied by maximal supersymmetry. These are difficult to establish in the absence of an off-shell supersymmetric formalism. In theories with less supersymmetry such protection is typically afforded to $F$ terms, which can be expressed as integrals over a subspace of the complete superspace. One might estimate the extent to which the derivative expansion of the four-graviton amplitude is protected by using on-shell superfield arguments. This is explicit in the pure spinor formalism of the superstring developed by Berkovits [5], in which terms of the form $\mathcal{S}^{(k)} R^{4}$ are $F$ terms for $k \leq 5$ and get vanishing contributions from $h>k$ loops. Such terms arise from integration over a subset of the 32 components of the left-moving and right-moving Grassmann spinor world-sheet coordinates, $\theta_{L}$ and $\theta_{R}$. As a result, the low-energy limits of both type IIA and type IIB superstring theories at $h$ loops have $\beta_{h}=h$ for $h=2,3,4,5$, and $\beta_{h} \geq 6$ for $h \geq 6$. Since $\mathcal{S}^{(h)} R^{4}$ terms are protected for $h \leq 5$ the $\mathcal{S}^{(6)} R^{4}$ interaction can only arise for $h \geq 6$. If we once more make the assumption that the power of $\Lambda_{d}$ does not increase in the process of taking the low-energy limit in $d$ dimensions, we can see from (5) that ultraviolet divergences are absent for the following cases:

$$
\begin{gathered}
d<2+18 / h, \quad h>5, \\
d<4+6 / h, \quad h=2, \ldots, 5 .
\end{gathered}
$$

This indicates that the nonrenormalization conditions in type II string theory [5] lead to the ultraviolet finiteness of the four-dimensional $N=8$ supergravity four-graviton amplitude up to at least eight loops $(h=8)$. Note that although types IIA and IIB four-graviton string theory amplitudes are equal only up to four loops $(h=4)$ in ten dimensions [5], they are identical at all loops in the $d$-dimensional low-energy supergravity limit.

One of the benefits of the superfield description of $F$ terms is that it includes all terms related by supersymmetry. For example, interactions involving higher powers of the curvature tensor of the form $\mathcal{S}^{(k)} R^{M}$, are $F$ terms if $2 k+$ $M<16$ [5] (where $\mathcal{S}^{(k)}$ is a monomial made of Mandelstam invariants of the $M$-particle amplitude). In this case an extension of the fermionic mode counting in [5] shows that there are no corrections beyond $h=$ $k+M-4$ loops. Generalizing our earlier analysis, this means that the $h$-loop $M$-graviton amplitude behaves as $\mathcal{S}^{(h+4-M)} R^{M}$ for $M \leq h+4$. It follows that the cutoff dependence of this amplitude is $\Lambda_{d}^{(d-4) h-6}$ for $h<4+$ $M / 2$ and $\Lambda_{d}^{(d-2) h-14-M}$ for $h \geq 4+M / 2$. Given the previous analysis, this would imply that the $M$-graviton amplitude is finite in four dimensions $(d=4)$ if $h<7+M / 2$. It is notable that these arguments suggest the absence of divergences that might have arisen according to various superspace arguments [12-15].

Finally, we return to the suggestion [4] that $\beta_{h}=h$. This was motivated by an indirect argument based on considerations of $M$-theory duality rather than direct string calculations and is therefore less well established. In particular, it is not yet apparent how this condition can be motivated by supersymmetry. In this case there is an extra power of $s, t$, or $u$ for every additional loop and the divergence of the $h$-loop integral is markedly reduced. Substituting $\beta_{h}=h$ in (5), and making the earlier smoothness assumption, it follows that ultraviolet divergences are absent when

$$
d<4+6 / h, \quad h \geq 2 .
$$

If correct, this would imply that ultraviolet divergences are absent to all orders in the four-graviton amplitude of fourdimensional maximal supergravity. Finiteness of the fourgraviton amplitude suggests finiteness of all $M$-point functions since they are interconnected by unitarity. Indeed, the arguments in [4] have an obvious extension to multigraviton amplitudes, which suggests that the $\mathcal{S}^{(k)} R^{M}$ interactions again have a dependence on the cutoff of the form $\Lambda_{d}^{(d-4) h-6}$. This leads to the same condition, (9), for ultraviolet finiteness of $M$-graviton amplitudes as in the fourgraviton case.

The bound (9) is the same as the condition for the absence of ultraviolet divergences in maximally supersymmetric Yang-Mills theory, which is known to be finite in four dimensions. Indeed, the work of [3] points to connections between loop amplitudes of maximal supergravity and those of maximal super Yang-Mills motivated in part by the Kawai-Lewellyn-Tye relations [16] that connect tree-level open and closed string theory. This suggests that $N=8$ supergravity may be more finite than previously expected $[17,18]$.

To summarize, in this Letter we have considered the implications for low-energy $d$-dimensional supergravity of the recently discovered nonrenormalization properties of higher-genus contributions to the four-graviton amplitude in type II superstring theory [5]. The relevant limit involves compactification of string theory on a $(10-d)$ torus followed by a low-energy expansion at fixed Newton constant $\kappa_{d}^{2}$. We argued that, subject to an important smoothness assumption, the four-graviton amplitude of $N=8$ super- 
gravity has no ultraviolet divergences up to at least eight loops. The nonrenormalization conditions were obtained in [5] by using the pure spinor formulation of string perturbation theory, in which $\mathcal{S}^{(h)} R^{4}$ is an $F$ term and gets no corrections beyond $h$ loops if $h \leq 5$. Similarly, we also argued that the $M$-graviton amplitude is ultraviolet finite when $h<7+M / 2$.

An important further issue that remains to be resolved is the fact that, in the low-energy limit under consideration, infinite towers of states in the nonperturbative sector of string theory (wrapped $D p$ branes, Neveu-Schwarz branes, Kaluza-Klein charges, and Kaluza-Klein monopoles) become massless. These are likely to give singular contributions that may cast doubt on the validity of the perturbative approximation, which only takes into account the perturbative states [19].

It would obviously be of interest if this understanding could be extended to derive the all-orders nonrenormalization conditions proposed in [4]. Interestingly, there are similar situations in highly supersymmetric theories in which an infinite number of higher-dimension operators are protected from renormalization even though a naive application of supersymmetry would suggest that only a finite number should be [20,21].

A priori, finiteness of $N=8$ seems very unlikely and, if true, would cry out for a natural explanation. One possible framework for such an explanation might be a variant of twistor string theory [22], which naturally describes $N=4$ Yang-Mills theory coupled to superconformal gravity $[23,24]$. Perhaps one of the proposals for a $N=8$ twistor string theory in [25] is on the right track.

We are grateful to Nathan Berkovits for many useful interactions and to Savdeep Sethi and Edward Witten for correspondence. We are also grateful to Hirosi Ooguri and John Schwarz for communications regarding the possible role of massless "nonperturbative" states. P. V. would like to thank the LPTHE of Jussieu for hospitality where part of this work was carried out. J. R. also acknowledges support by MCYT FPA No. 2004-04582-C02-01. This work was partially supported by the RTN Contracts No. MRTN-CT2004-503369, No. MRTN-CT-2004-512194, and No. MRTN-CT-2004-005104, and by the ANR Grant No. BLAN06-3-137168.

[1] E. Cremmer and B. Julia, Phys. Lett. B 80, 48 (1978); B. de Wit and H. Nicolai, Nucl. Phys. B208, 323 (1982).
[2] E. Cremmer, B. Julia, and J. Scherk, Phys. Lett. B 76, 409 (1978).

[3] Z. Bern, L. J. Dixon, D. C. Dunbar, M. Perelstein, and J. S. Rozowsky, Nucl. Phys. B530, 401 (1998).

[4] M. B. Green, J. G. Russo, and P. Vanhove, J. High Energy Phys. 02 (2007) 099.

[5] N. Berkovits, hep-th/0609006.

[6] M. B. Green and J.H. Schwarz, Phys. Lett. B 109, 444 (1982).

[7] S. Weinberg, Phys. Rev. 140, B516 (1965); D. C. Dunbar and P.S. Norridge, Classical Quantum Gravity 14, 351 (1997).

[8] M. B. Green, J.H. Schwarz, and L. Brink, Nucl. Phys. B198, 474 (1982).

[9] V. A. Smirnov, Phys. Lett. B 460, 397 (1999); J. B. Tausk, Phys. Lett. B 469, 225 (1999); C. Anastasiou, J. B. Tausk, and M.E. Tejeda-Yeomans, Nucl. Phys. B, Proc. Suppl. 89, 262 (2000).

[10] E. D'Hoker and D. H. Phong, Nucl. Phys. B715, 3 (2005); N. Berkovits and C. R. Mafra, Phys. Rev. Lett. 96, 011602 (2006); N. Berkovits, J. High Energy Phys. 01 (2006) 005.

[11] M. B. Green and P. Vanhove, J. High Energy Phys. 01 (2006) 093.

[12] P. S. Howe and U. Lindstrom, Nucl. Phys. B181, 487 (1981).

[13] P. S. Howe, K. S. Stelle, and P. K. Townsend, Nucl. Phys. B236, 125 (1984).

[14] P. S. Howe and K. S. Stelle, Int. J. Mod. Phys. A 4, 1871 (1989).

[15] P. S. Howe and K. S. Stelle, Phys. Lett. B 554, 190 (2003).

[16] H. Kawai, D. C. Lewellen, and S. H. H. Tye, Nucl. Phys. B269, 1 (1986).

[17] Z. Bern, N. E. J. Bjerrum-Bohr, and D. C. Dunbar, J. High Energy Phys. 05 (2005) 056; N. E. J. Bjerrum-Bohr, D. C. Dunbar, and H. Ita, Phys. Lett. B 621, 183 (2005); N. E. J. Bjerrum-Bohr, D. C. Dunbar, H. Ita, W. B. Perkins, and K. Risager, J. High Energy Phys. 12 (2006) 072.

[18] Z. Bern, L. J. Dixon, and R. Roiban, Phys. Lett. B 644, 265 (2007).

[19] Similar observations have independently been made by Hirosi Ooguri and John Schwarz.

[20] S. Sethi and M. Stern, J. High Energy Phys. 06 (1999) 004; S. Sethi, J. High Energy Phys. 10 (2004) 001.

[21] M. Dine and J. Gray, Phys. Lett. B 481, 427 (2000).

[22] E. Witten, Commun. Math. Phys. 252, 189 (2004).

[23] N. Berkovits, Phys. Rev. Lett. 93, 011601 (2004).

[24] N. Berkovits and E. Witten, J. High Energy Phys. 08 (2004) 009.

[25] M. Abou-Zeid, C. M. Hull, and L. J. Mason, hep-th/ 0606272. 